Supporting information for

\title{
On-surface Self-Assembled Structural Transformation Induced by Schiff Base Reaction and Hydrogen bonds
}

Peng Lei, ${ }^{a, b *}$ Jing-fei Hou, ${ }^{a, b *}$ Yu-chuan Xiao, ${ }^{a, b *}$ Feng-ying Zhao,${ }^{c^{*}}$ Xiao-kang Li, ${ }^{d^{*}} \mathrm{Ke}$ Deng $^{a^{*}}$ Qing-dao Zeng, ${ }^{a, b}$ *

${ }^{a}$ CAS Key Laboratory of Standardization and Measurement for Nanotechnology, CAS Center for Excellence in Nanoscience, National Center for Nanoscience and Technology (NCNST), Beijing 100190, China E-mail: zengqd@,nanoctr.cn, kdeng@nanoctr.cn

${ }^{b}$ Center of Materials Science and Optoelectonics Engineering, University of Chinese Academy of Sciences, Beijing 100049, China.

c Jiangxi College of Applied Technology, Ganzhou 341000, China E-mail: fengying2599@sina.com

${ }^{d}$ College of Chemistry and Chemical Engineering, Gannan Normal University, Ganzhou, Jiangxi 341000, PR China E-mail: gnnulxk@163.com

Number of pages: 5

Number of figures: 2

1. The self-assembled networks of TCDB template molecules

2. The self-assembled molecular morphology of BAPTT-AIPA without the TCDB template

3. Benchmark of accuracy of DFT calculations 


\section{The self-assembled networks of TCDB template molecules}

Figure S1 show the self-assemblies of TCDB molecules. The bright spots correspond to the benzene cores of TCDB molecules, and the chains are corresponding to the alkyl chains attached to the benzene cores. Two TCDB molecules form a dimer by four pairs of hydrogen bonds between four carboxyl acids from two molecules, respectively. The opposite TCDB molecules in one dimer thus form a cavity. Combined with the van der Waals interactions between the alkyl chains, the TCDB molecules finally form the twodimensional tetragonal networks on the highly oriented pyrolytic graphite (HOPG) surface. The unit cell is labelled in Figure S1b, which contains two TCDB molecules. The parameters of the unit cell are as follows: $a=4.2 \pm 0.1 \mathrm{~nm}, \mathrm{~b}=2.1 \pm 0.1 \mathrm{~nm}$, and the angle $\alpha$ between two directions is about $69.8 \pm 1^{\circ}$. The molecular models of TCDB networks are shown in previous study. ${ }^{1}$

The cavities of TCDB self-assembled networks could be utilized as a template for the two-dimensional host-guest architectures. Thus, the functional molecules with appropriate size could be trapped into the cavities. The parameters of the TCDB cavity are as follows: $\mathrm{x}=2.2 \pm 0.1 \mathrm{~nm}, \mathrm{y}=1.4 \pm 0.1 \mathrm{~nm}$. In this work, the sizes of molecule Benzaldehyde, 4,4',4",4'"- (21H,23H-porphine-5,10,15,20-tetrayl) tetrakis(BAPTT) and molecule Tetra-(4-aldehyde benzene) ethylene (TABE) are less than 2 $\mathrm{nm}$, and can form the host-guest architectures with the template molecule TCDB. While the size of molecule Tetra-(4-aldehyde - (1,1-biphenyls)) ethylene (TABPE) is larger than the cavity of TCDB networks, and cannot form the host-guest architectures. 

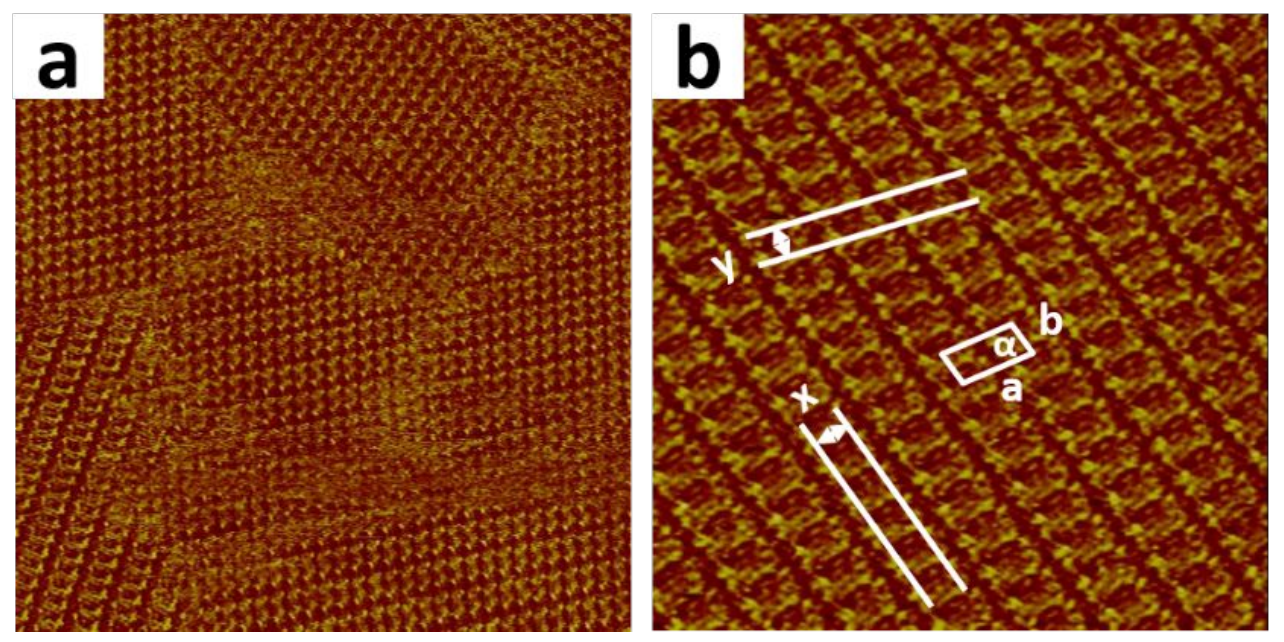

Figure S1. (a) The large-scale STM image of the self-assembled TCDB networks on the phenyl octane/HOPG surface. The tunneling conditions are as follows: $I_{\text {set }}=277.7$ $\mathrm{pA}, V_{\text {bias }}=654.3 \mathrm{mV}$. The scan size is $73 \mathrm{~nm} \times 73 \mathrm{~nm}$; (b) The high-resolution STM image of the TCDB molecular networks. The tunneling conditions are as follows: $I_{\text {set }}=$ $338.7 \mathrm{pA}, V_{\text {bias }}=-379.6 \mathrm{mV}$. The scan size is $31 \mathrm{~nm} \times 31 \mathrm{~nm}$, and the unit cell is labelled in the image.

2. The self-assembled molecular morphology of BAPTT-AIPA without the

\section{TCDB template}

We have examined the reaction process of molecule BAPTT with the active reactant 5-AIPA, and obtained the self-assemblies of the on-surface reaction product BAPTTAIPA molecules at the HA/HOPG surface with the help of TCDB template. Meanwhile, we also investigate the reaction product molecular self-assemblies without the TCDB template. Figure S2a and S2b correspond to the large-scale and high-resolution STM image of the reaction product molecular self-assemblies, respectively. The X-shaped 
molecular morphologies are similar with that on the TCDB template, and the molecular size was measured to be about $2.4 \mathrm{~nm}$, which is nearly the same size as the molecule BAPTT-AIPA. Thus, we speculated that the BAPTT-AIPA molecules were formed at the HA/HOPG surface via the condensation reaction between the aldehyde and the amine of 5-AIPA. Although we have not obtained the molecular self-assemblies of BAPTT, as the aromatic rings are not coplanar. After we performed the on-surface condensation reaction, we still gained the self-assembled molecular architectures of BAPTT-AIPA as same as that with the TCDB template.
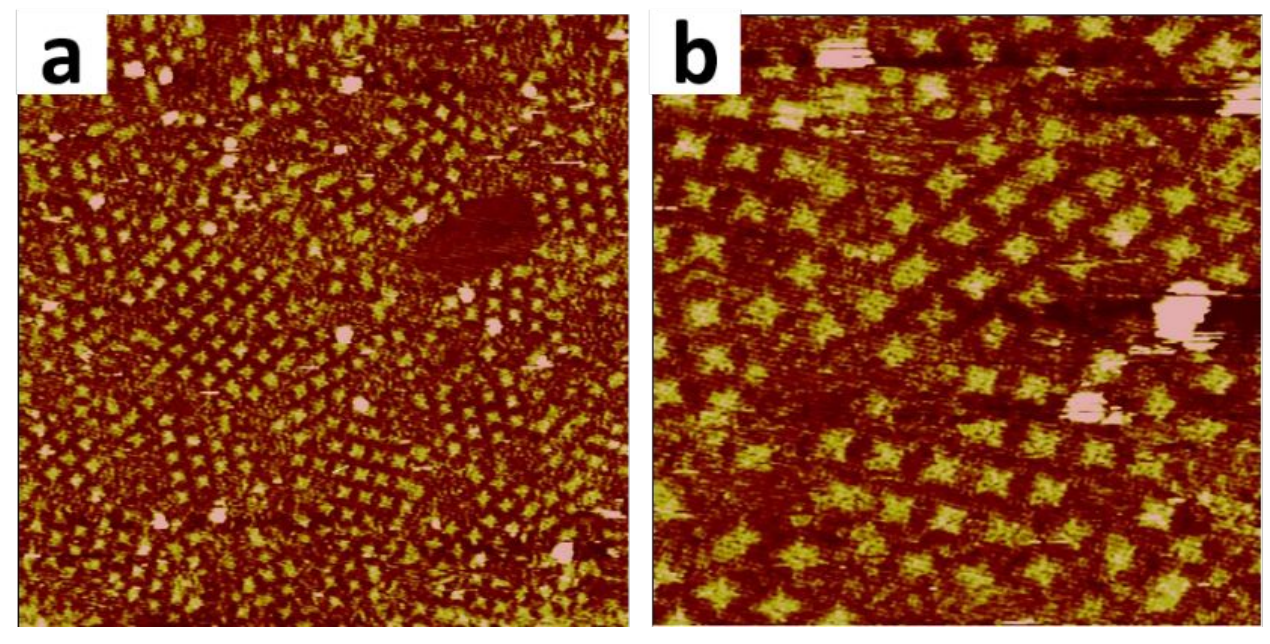

Figure S2. (a) The self-assembled large-scale STM image of the synthesized BAPTTAIPA molecules without the TCDB template at 120 mins on the HA/HOPG surface. Scan size: $80.3 \mathrm{~nm} \times 80.3 \mathrm{~nm}$. The tunneling conditions are as follows: $I_{\text {set }}=289.9 \mathrm{pA}$, $V_{\text {bias }}=525.5 \mathrm{mV}$. (b) The high-resolution STM image of BAPTT-AIPA molecules without the TCDB template at 120 mins on the HA/HOPG surface. Scan size: $30.9 \mathrm{~nm}$ $\times 30.9 \mathrm{~nm}$. The tunneling conditions are as follows: $I_{\text {set }}=289.9 \mathrm{pA}, V_{\text {bias }}=525.5 \mathrm{mV}$. 


\section{Benchmark of accuracy of DFT calculations}

To evaluate the accuracy of the DFT calculations, we have calculated the interaction between benzene and graphite, and the interaction between n-pentane and graphite as a benchmark. The interaction between benzene and graphite is about $-10.69 \mathrm{kcal} \mathrm{mol}^{-1}$, which agrees well with the experimental measurements $\left(9.7 \sim 10 \mathrm{kcal}^{\mathrm{mol}^{-1}}\right.$ at $\left.\mathrm{T}=298 \mathrm{~K}^{2-4}\right)$. The interaction between $\mathrm{n}$-pentane and graphite is about $-10.70 \mathrm{kcal} \mathrm{mol}^{-}$

${ }^{1}$, which is also in very good agreement with the theoretical formula $\left(-10.15 \mathrm{kcal} \mathrm{mol}^{-1}\right)$ for the adsorption energy of n-alkanes on the graphite surface derived in previous papers $^{5,6}$ in this series: $-\Phi=0.9+1.85 \mathrm{n} \mathrm{kcal} \mathrm{mol}^{-1}$. It indicates that our results with DFT calculations are reasonable.

\section{Reference:}

1. Kong, X.-H.; Deng, K.; Yang, Y.-L.; Zeng, Q.-D.; Wang, C., H-Bond Switching Mediated Multiple Flexibility in Supramolecular Host-Guest Architectures. J. Phys. Chem. C 2007, 111, 17382-17387.

2. Isirikyan, A. A.; Kiselev, A. V. J. Phys. Chem. 1961, 65, 601.

3. Pierotti, R. A.; Smallwood, R. E. J. Colloid Interface Sci. 1966, 22, 469.

4. Zacharia, R.; Ulbricht, H. and Hertel T., Phyiscal Review B 2004, 69, 155406.

5.Avgul, N. N.; Berezin, G. I.; Kiselev, A. V. and Lygina, I. A. L Phys. Chem. 1956, 30, 2106.

6.Avgul, N. N. and Kiselev, A. V. Proc. Acad. Sci. USSR 1957,112, 673. 